\title{
Integrating Economic Parameters and Engineering Parameters in Design and Management of Hydraulic Systems
}

\author{
Zohrab Samani, Jorge Garcia \\ New Mexico State University/City of Las Cruces
}

Traditionally, pumps are designed based on engineering parameters while economic analysis is often limited to cost estimate after the design. This paper introduces the concept of economic efficiency and its implication in design and management of pumping systems. The paper describes examples in which economic parameters play an important role in the design and management of the engineering systems.

The traditional approach in designing a pump has been to select a pump which can provide the required discharge and head as closely as possible and at the same time provide a good pump efficiency. A good pump efficiency does not necessarily reduce energy cost unless it is accompanied by appropriate management plan (Weighing, 1). In studying the benefits of improving pumping efficiency, Hanson(2), concluded that "growers should not expect energy saving from improved pumping efficiency unless the actual horsepower demand is reduced". In designing pumping plants, Economic analysis has often been limited to calculation of operating cost and capital cost based on the engineering parameters. This type of designs are based on the assumption of steady-state operational condition. However, pumps may operate under variable hydraulic conditions. Under variable operational conditions, the ultimate cost of a system will depend on the combined effect of design,operation and economic parameters. It is therefore necessary to include the management and economic parameters during the actual design of the system instead of trying to remedy the situation after the fact.

The following discussion describes the difference between mechanical efficiency and economical efficiency and the importance of the economic parameters in the design and management of pumping plants.

The economical efficiency (ECE) is defined as the amount of liquid pumped per unit of energy used in the process. 
If a pump operates for a specific period of time (T) and pumps at a specific discharge rate of $(\mathrm{Q})$, the weight of liquid which is lifted can be described as

In which;

$W=\rho Q T$

$\mathrm{W}=$ the total weight of the pumped liquid $(\mathrm{W})$

$\rho=$ density of the fluid $\left(\mathrm{M} / \mathrm{L}^{3}\right)$

$\mathrm{Q}=$ Discharge rate $\left(\mathrm{L}^{3} / \mathrm{T}\right)$

$\mathrm{T}=$ duration of operation

The amount of energy which is used to pump the above described liquid is (karassik et al, 3):

in which:

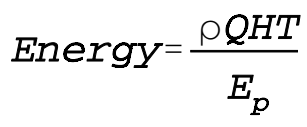

$\rho=$ fluid density $\left(M / L^{3}\right)$

$\mathrm{Q}=$ discharge $\operatorname{rate}\left(\mathrm{L}^{3} / \mathrm{T}\right)$

$\mathrm{H}=$ head against which the liquid is lifted (L)

$\mathrm{T}=$ duration of pumping

$\mathrm{E}_{\mathrm{p}}=$ pump efficiency

The economical efficiency is then calculated by the ratio of equation (1) to equation (2) as:

$$
E C E=\frac{E_{p}}{H}
$$

in which:

$\mathrm{ECE}=$ economical efficiency which represents the amount of liquid lifted per unit of energy used.

The following example describes the effect of variable operational conditions on the mechanical and economical efficiency of a pump. A centrifugal pump operation fluctuates between 65 and 55 liters per second. At $60 \mathrm{~L} / \mathrm{S}$, the operational head is $8 \mathrm{~m}$ and pump efficiency is 75 percent. At $55 \mathrm{~L} / \mathrm{S}$, the operational head is $12 \mathrm{~m}$ and pump efficiency is 80 percent. The optimum pump efficiency is obtained when the pump is operating at $55 \mathrm{~L} / \mathrm{S}$. However, from economical point of view, the $55 \mathrm{~L} / \mathrm{s}$ operation point is not the optimum point. The economical efficiency (ECE) at 55 
$\mathrm{L} / \mathrm{S}$ is equal to 6.67 while the ECE at $60 \mathrm{~L} / \mathrm{S}$ is 9.38 which is a difference of 40 percent in energy cost while the pump efficiency varies only 5 percent. From a design point of view, one may not worry about a 5 percent variation on efficiency or 9 percent variation on discharge rate, but a 40 percent variation in cost could be significant. Therefore, this may not be the right pump for this condition. The optimum design and operation for this condition is the one which will maximize the integral effect of economical efficiency over the specified time period as:

in which: $\quad \operatorname{Max}$. Benefit $=\operatorname{Max} \cdot \int_{t 1}^{t 2}\left(\frac{H}{E}\right) d T$

dT represents the increment of time, and

$\mathrm{t} 1$, and $\mathrm{t} 2$ represent the period of pump operation.

The combined effect of design, economic and management can become even more complex in systems with multiple pumps and pipelines. Table 1, represents the hydraulic characteristics of 3 pumps which supply water to the pipe network at New Mexico State University (NMSU). The network consists of several hundred pipes and a 4 million gallon tank. In this system, the discharge rate and mechanical efficiency of each individual pump does not vary significantly (Table 1). However, there is significant variation in economical efficiency of each pump.

Table 1. Operational Characteristics of NMSU Hydraulic System

\begin{tabular}{lllll} 
Pump ID \#. & $\mathrm{H}(\mathrm{m})$ & $\mathrm{Q}(\mathrm{L} / \mathrm{S})$ & $\mathrm{E}$ & ECE (E/H) \\
\hdashline 1 & 80.8 & 31.5 & 81 & 1.00 \\
10 & 109.2 & 97.7 & 81.5 & 0.75 \\
14 & 142.5 & 132.3 & 81.5 & 0.57
\end{tabular}

The disparity in the economical efficiencies of these pumps is an example of the significant difference which can occur in operation cost, where pumps are designed based on engineering factors alone.

The sequence of operation of these pumps at NMSU is also another example of lack of attention to economical efficiency. Currently these pumps operate based on the water level at the tank. When water level at the tank drops by about 1 meter, the pump\# 14 is turned on first, followed by pump \#10 if the water level drops by 1.5 meter and ultimately pump \# 1 if the water level drops further. At first glance, nothing appears to be wrong with this sequence of operation since all three pumps are running at peak mechanical efficiency. If the sequence of operation is reversed however, there could be significant cost saving in this operation. Currently, NMSU is monitoring the electric power used by each pump to determine the economic implications of alternative operations of this system. 


\section{CONCLUSION}

Traditional approach in design and management of pumping system solely based on engineering parameters, may result in systems which are mechanically efficient but economically inefficient. A term called economical efficiency is defined which can be used for optimum design and management of pumping plants. The objective of using economical efficiency is to design and manage systems which are hydraulically sound and economically efficient.

1. Weighing W. Energy Efficient Irrigation Practices Handbook. University of Idaho, Energy Division, 1996.

2. Hanson, B. Benefits and Costs of Improving Pumping Efficiency. California Agriculture, July-August 1988.

3. Karassik, I. J., W.C. Krutzch, W.H. Fraser and J.P. Messina. Pump Handbook.New York, Mcgrow Hill Pub. Co., 1996.

Zohrab Samani is Associate Professor at the Civil Engineering Department at New Mexico State University, Las Cruces, NM. His area of research and teaching include; water resources development, groundwater hydrolog and hydraulic systems.

Jorge Garcia is Chief Utility Engineer with the City of Las Cruces Utility Department. He is in charge of design and management of municipal water system for the City of Las Cruces, NM. 